\title{
V2I Applications in Highways: How RSU Dimensioning Can Improve Service Delivery
}

\author{
Georgios Charalampopoulos, Tasos Dagiuklas \\ School of Science \& Technology \\ Hellenic Open University \\ Patras, Greece \\ Email :\{gcharalampopoulos, dagiuklas\}@eap.gr \\ http://cones.eap.gr
}

\author{
Theofilos Chrysikos \\ Dept of Electrical and Computer Engineering \\ University of Patras \\ Patras, Greece \\ Email: txrysiko@ece.upatras.gr
}

\begin{abstract}
This paper investigates the performance of Vehicle-to-Infrastructure (V2I) services over Vehicular Networks (VANETs) that are assisted by Road Side Units (RSU). More specifically, an analytical study of RSU dimensioning and a respective module is designed and developed in a simulated VANET environment. Two V2I application scenarios (e.g. car crash, spot weather) are considered in order to evaluate the impact of RSUs, vehicles' size and speed and car crash start time and duration on applications' performance. It is shown that the VANET network metrics (Packet Loss and Packet Delivery Ratio) are affected by the available MAC Bit rates and application scenarios. Mobility model metrics (Total Busy Time and Total $\mathrm{CO} 2$ Emissions) are also affected by the different application scenarios, number and type of vehicles.
\end{abstract}

Keywords-VANETs; RSU Deployment; V2I Communication; Warning Services

\section{INTRODUCTION}

There is a growing interest in deployment of value-added services in Vehicular Networks (VANETs). These networks can be used for a variety of applications such as traffic management, speed reduction / work zone warning and spot weather impact warning. Until recently, research has been mainly focused on approaches to employ advanced VehicularTo-Infrastructure (V2I) and Vehicular-To-Infrastructure (V2V) technologies. Particular emphasis is given to critical applications in order to provide safety messages (i.e. warning messaging to drivers for the weather, traffic management, collisions etc) [1].

Amongst others, the IEEE $802.11 \mathrm{p}$ protocol is specifically developed for vehicular communications and its specifications take into consideration both multipath phenomena and Doppler shift in order to provide reliable communications for intra-vehicle and vehicle-to-central infrastructure [2], [3]. A number of research works [4]-[10] are investigated the capability and robustness of $802.11 \mathrm{p}$ standardization and are provided solution for various issues related to vehicular communications. The specific impact of wireless channel characterization and the investigation of deterministic, distance dependent losses as well as stochastic losses, expressed as the 'excess path loss' [11], are also studied [12] [13]. Furthermore, LTE networks [14] are considered for Public Safety networks for vehicular users [15]-[17]. Whereas these works focus on urban areas and macro/micro-cell scenarios, their findings can also be employed for any vehicular scenario, as long as the intrinsic channel and topology characteristics are taken into consideration.

Road Side Units (RSUs) can provide communication between vehicles and infrastructure and act similar to wireless LAN access points. Moreover, RSUs must be able to allocate channels to OBUs (vehicular On-Board Units) if required and to provide services to Public Safety OBUs that are mainly utilized in vehicles of police, fire trucks and ambulances in emergency situations [28].

Unlike previous research works, the main goal of this paper is to specify RSU dimensioning in VANETs by taking into account the road's size and the number of vehicles and investigate the impact of RSUs in order to improve the V2I applications along highways. The aim of RSUs is to assist VANETs and enhance the delivery of V2I warning messaging services regarding the public safety in highways. Two warning applications have been developed in a simulated environment allowing the creation of warning messages. The first one regards the warning generation after a single crash/collision between two vehicles has occurred. The second one refers to a spot weather warning application. Important parameters of these applications are the vehicle density, different types of vehicles and MAC Bit rates.

The aim is to investigate the impact of these applications on the mobility models and how RSUs affect network performance because most of the Intelligent Transport Systems (ITS) safety applications are based on information exchange between different communication types of VANETs (e.g. V2V,V2I and V2X) in order to get an up-to-date view of an emergency situation [30]. Moreover, we study the impact of the vehicles' size and car crash duration involved in the collision on the performance of VANET using a bigger road network and high number of vehicles in comparison with previous research works.

The paper is structured as follows: the Road Side Unit deployment is described in Section II. VANET map enhancements and mobility modeling with RSU for improving ITS are presented in section III. The results are discussed in section IV and conclusions are presented in section V. Section VI addresses issues that will be part of immediate future work in order to investigate more solutions that can be employed in the field of RSU dimensioning. 


\section{ROAD SIDE UNIT (RSU) DEPLOYMENT}

Our work focuses on the RSU deployment for a highway scenario based on the sub-optimal conditions. This topology varies significantly from the macro-micro cell conditions examined in urban deployment scenarios, where an obstacledense environment and severe scattering affect signal propagation. In a highway scenario, a dominant Line-of-Sight (LOS) component is expected, with the appropriate selection of transmitter antenna height. Therefore, the average path loss can be estimated in reliable manner by the deterministic distance-dependent attenuation losses [18]. The local mean value of the received signal power can be estimated from the transmitter characteristics and the average path loss, and the rapid fluctuations of the signal amplitude around this local mean value are known to follow the Rice distribution [19].

When distance-dependent attenuation is considered, in the presence of a dominant LOS component, the average path loss (in $\mathrm{dB}$ ) is provided by the following formula [18]:

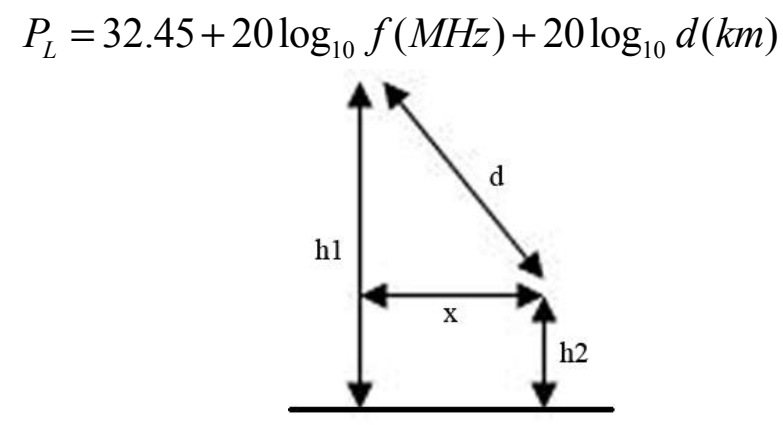

Fig. 1. Precise calculation of T-R separation

The actual transmitter-receiver distance (T-R separation) was calculated on the basis of Fig.1, by considering a properly scaled map of the area as well as GPS data. Actual antenna heights are considered therefore our calculations are conducted on the basis of a 3D channel model [19]. Transmitter antenna height was assumed to be 7 meters, whereas a height of 3 meters was considered for the receiver antenna.

The elevated height of the transmitter antenna ensures that there is a seemingly undisrupted LOS component for the whole area of coverage of each RSU [20]-[22]. The deployment of the RSU takes place by the side of the left lane. Each lane is assumed to be 9 meters wide. A highway length of 6 kilometers is examined in this case study. Therefore, a specific received power (local mean value) threshold must be selected in order to determine the coverage area of each RSU with regard to area-edge "boundary conditions".

Since the receiver sensitivity level for the IEEE $802.11 \mathrm{p}$ protocol is at $-89 \mathrm{dBm}$ [2]-[3], a $4 \mathrm{~dB}$ fade margin is allowed for small-scale multipath phenomena, therefore a threshold of $-85 \mathrm{dBm}$ is selected as a boundary condition for the edge of the coverage area of each RSU. An Omni-directional antenna is considered for both RSU and the VANET receiver. A total Effective Isotropic Radiated Power (EIRP) of $10 \mathrm{~mW}$ (10 $\mathrm{dBm}$ ) is considered [2]-[3] for the transmitting RSU.
A $2 \mathrm{~dB}$ gain is considered for the receiving antenna, counterbalanced by $2 \mathrm{~dB}$ of excess path loss, defined by Jakes as "the difference (in decibels) between the computed value of the received signal strength in free space and the actual measured value of the local mean received signal" [11].

Therefore the total average path loss is provided by:

$$
L_{\text {total }}=P L\left(d_{0}\right)+N \log _{10}\left(d / d_{0}\right)+X_{\sigma}
$$

Where $P L\left(d_{0}\right)$ is the path loss at the reference distance, equal to $1 \mathrm{~m}, N=10 n$ is the slope factor (where $n$ is the path loss exponent) and $X_{\sigma}$ is the zero-mean Gaussian random variable standing for the excess path loss (in $\mathrm{dB}$ ).

The excess path loss attributed to losses caused by taller vehicles (trucks, buses etc) and they follow the Gaussian (normal) distribution (logarithmic values), the Probability Density Function (PDF) of which is given by [18]:

$$
p(x)=\frac{1}{\sigma \sqrt{2 \pi}} e^{-\frac{(x-\bar{x})^{2}}{2 \sigma^{2}}}
$$

Where $x$ is the received power (logarithmic value) in each measurement location (local mean strength), $\bar{x}$ is the average received power (logarithmic value) for all measurement locations (mean value of the received power overall the topology in question), and $\sigma$ is the standard deviation of the excess path loss (in $\mathrm{dB}$ ).

Our calculations provide a critical distance of 213 meters as the radius of each Omni-directional RSU antenna. Therefore each RSU covers a total length of 426 meters along the total length of 6 kilometers of the highway section examined in this work. Overall, 15 RSU will be needed to cover a highway length of $6 \mathrm{Km}$ and width of 18 meters.

The actual local mean value of the received signal strength as well as the subsequent Signal-to-Noise ratio (SNR) depend on the location of the vehicle with respect to the serving RSU, the velocity, and the relative position of the vehicles for different scenarios. Our assumption for a $2 \mathrm{~dB}$ excess path loss indicates an averaging of losses caused by other vehicles interfering with the signal path. A receiver antenna height of 3 meters has been taken into consideration for the RSU dimensioning. As the vehicles in question move further away from the RSU towards the boundary of each area covered by each RSU (7668 square meters), the other vehicles meddle with the signal path. However, the choice of a 7-meter height for the mounting position of the RSU provides a less time variant channel and for the most part, LOS is established.

The robustness of this dimensioning scenario has been validated in [22] for the specific antenna height. Our consideration of the additional $2 \mathrm{~dB}$ of excess path loss includes all worst-case probable losses, depending on variable 
vehicle density and relative position throughout the topology in question. As confirmed in [22], the driving direction holds no impact for the said mounting height.

Since a $-85 \mathrm{dBm}$ threshold value is considered for the local mean value of the received signal power and noise levels assumption to obtain the generically considered $-100 \mathrm{dBm}$ value [18], the SNR of the vehicle in question adopts the threshold of $15 \mathrm{~dB}$. This value is in accordance with the required SNR threshold for achieving data rates up to 18 Mbps, for a vehicle velocity of $120 \mathrm{~km} / \mathrm{h}$ (approximately 34 $\mathrm{m} / \mathrm{s}$ ). It also greatly exceeds the SNR requirements for the packet length for the same velocity and RSU height conditions [22]. Analytical simulation scenarios are provided in the following section.

\section{VANET MAP ENHANCEMENTS AND MOBILITY MODELING WITH RSU FOR IMPROVING ITS}

\section{A. Mobility Model}

For the VANET simulation scenarios, realistic mobility models in microscopic simulation (it takes into account road and node characteristics) are used providing road maps, the number of travelling cars and some road and car parameters such as maximum car speed, road limitations, departure and arrival times of each car [23]. JOSM (Java OpenStreetMap Editor) [24] is used to modify the map for the simulation scenarios [25].

Using the filter dialog in JOSM, certain types of objects are temporarily hidden from display, allowing easier editing in crowded areas. More specifically, for our simulation scenarios the map is edited using filters allowing to hide, disable or select specific groups of objects based on flexible custom parameters and only the highway is kept.

An important factor regarding the RSU dimensioning is the length and the distance of the highway's road network. A parser is created to calculate the road network that will be used in accordance with the street map to place the RSUs in the map and to carry out the simulations [18]. This parser also is used to calculate the number of vehicles for the five different node densities that are used for the simulation scenarios below.

Two vehicle types (vTypes) are used for the simulation scenarios to define the vehicle's purely physical parameters studying the impact of these in VANET network statistics. Moreover, different vehicle types can be used in the same scenario to represent different type of vehicles such as cars, taxis, buses and emergency vehicles for example police cars or fire trucks giving higher values in maxSpeeds and priorities [26].

\section{B. Simulation Scenarios}

For Vehicle Crash and spot weather impact warning application simulation, the Veins framework [28] is used because is integrates the OMNeT++ and SUMO [27] network simulator and can offer Vehicle to RSU communication. The Veins framework version for V2I includes IEEE 802.11p and IEEE 1609.4 DSRC/WAVE network layers, supporting realistic maps and traffics. The IEEE 1609.4 defines the multichannel and QoS operation of radios; vehicles with a single radio will periodically switch among multiple channels [28].

Firstly, a realistic map and the road network are imported from OpenStreetMap.com [25], the routes of the cars are generated using SUMO and then exported to the network simulator. OMNeT++ considers all the cars and RSUs as nodes and simulates the scenario. Veins can modify the scenario in SUMO and the realistic traffic of the highway is simulated as a VANET in OMNeT++. The Simulation scenario is configured for a dataset of 1000 seconds. Five different node densities are used with 108 (D1), 216 (D2), 324 (D3), 648 (D4) and 972 (D5) vehicles on the map under study. Each scenario is replicated 20 times. Moreover, the route $\mathrm{xml}$ files are modified changing the number of vehicles to represent the different node densities and finally, two different types of vehicles are set, 3.5 meters (vT1) for small and 5.5 meters (vT2) for medium vehicles with a maximum speed of $35 \mathrm{~m} / \mathrm{s}$, evaluating how the different type of vehicles affect the simulation results.

Two different simulation scenarios are created with a car crash and spot weather impact warning messaging. Both messages have a length of 32 bytes. All the simulation scenarios run with two different vehicle types (vT1 or vT2), five different vehicle densities (D1, D2, D3, D4 or D5) and 6 or $18 \mathrm{Mbps}$ MAC Bit rate. For each scenario, there is a different start time and duration of 200 seconds for the vehicle crash and five different locations (spots) in the road network for Weather Warning Messaging. The network parameters for our simulations are MAC Transmission Power $=20 \mathrm{~mW}$, Physical Thermal Noise $=-110 \mathrm{dBm}$ and Minimum Carrier Frequency $=5.89 \mathrm{GHz}[28]$.

Finally, taking into account the dimensioning described in Section II concerning the RSU deployment, 15 RSUs deployed across 6 kilometers of the highway in question, will be used for the simulation.

\section{RESULTS}

The results from the simulation scenarios are presented in the following graphs. More specifically, Packet Loss, Packet Delivery Ratio (PDR), Total Busy Time and Total CO2 Emissions will be discussed regarding the V2I network and the mobility model. Our goal is to evaluate how these metrics are affected by different applications, node densities and vehicle types. Fig. 2 and 3 show the PDR for different VANET V2I Applications and how this metric is influenced by the different node densities and Bit rates.

For Car Crash Warning Application and 108 vehicles, the PDR's highest value is $91.27 \%$ for $6 \mathrm{Mbps}$ and $92.33 \%$ for $18 \mathrm{Mbps}$ Bit rates respectively. Comparing the scenarios with same number of vehicles and different application, the PDR is $92.41 \%$ for $6 \mathrm{Mbps}$ and $92.73 \%$ for $18 \mathrm{Mbps}$ Bit rate. For D4 and D5, PDR is $98.64 \%$ and $99.97 \%$ for $6 \mathrm{Mbps}$ Bit rate and $99.9857 \%$ and $99.9868 \%$ respectuvely for $18 \mathrm{Mbps}$. For both Warning Messaging Applications, the PDR increases when the number of vehicles increases due to the fact that more and more vehicles close to the area of interest must be updated for the warning events. 


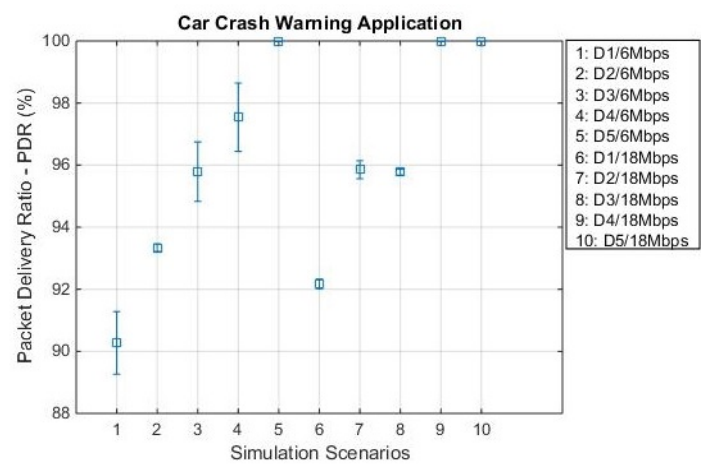

Fig. 2. Packet Delivery Ratio for Car Crash Warning Appication

A higher density of vehicles suggests that the statistical distribution of the vehicles throughout the topology covered by each RSU will be such, that an optimal route will be selected as the signal traverses from the roadside unit to the vehicular receiver. The different heights of vehicles do not meddle with the signal path since a 'high RSU' [22] scenario has been employed, providing a sufficient RSU antenna height so that obstruction losses do not exceed $2 \mathrm{~dB}$ (with a probability of $95 \%$ ).

Broadcast messages are used for sending emergency warning messages and periodically broadcasting vehicle state information. Moreover, the MAC Bit rate, from 6 to $18 \mathrm{Mbps}$, also affects the VANET V2I network for both applications because Packet Delivery Ratio has higher values for the scenario with $18 \mathrm{Mbps}$ Bit rate than the scenario with $6 \mathrm{Mbps}$, as seen in the respective graphs.

Fig.4 and 5 depict the Packet Loss, investigating how different warning applications, node densities and MAC Bit rates affect this network metric. The rate of packet loss is lower for the Spot Weather than the Car Crash warning.

For the application with Car Crash and $6 \mathrm{Mbps}$ Bit rate, in Packet Loss the highest values are $52.22 \%, 35.92 \%$ and $32.64 \%$ for 108,216 and 324 vehicles respectively. For the same application and $18 \mathrm{Mbps}$ Bit rate, the highest values are $44.05 \%$ for $108,30.37 \%$ for 216 and $24.63 \%$ for 324 vehicles. Moreover, for the highest value of vehicles, the highest value of packet loss for $6 \mathrm{Mbps}$ Bit rate is $19.08 \%$ and for $18 \mathrm{Mbps}$ is $0.329 \%$.

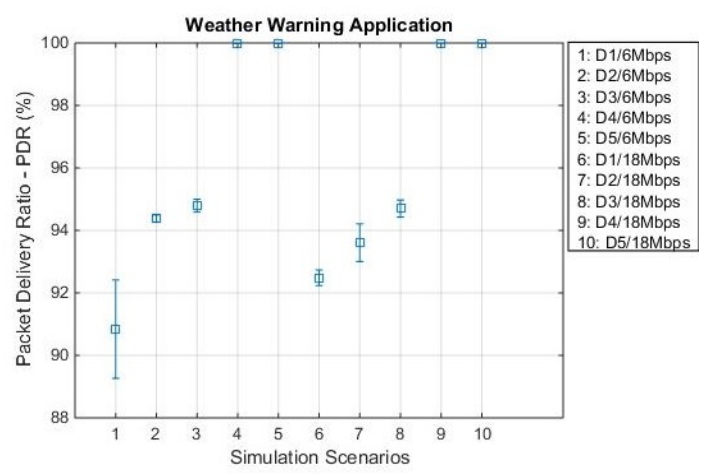

Fig. 3. Packet Delivery Ratio for Weather Warning Appication

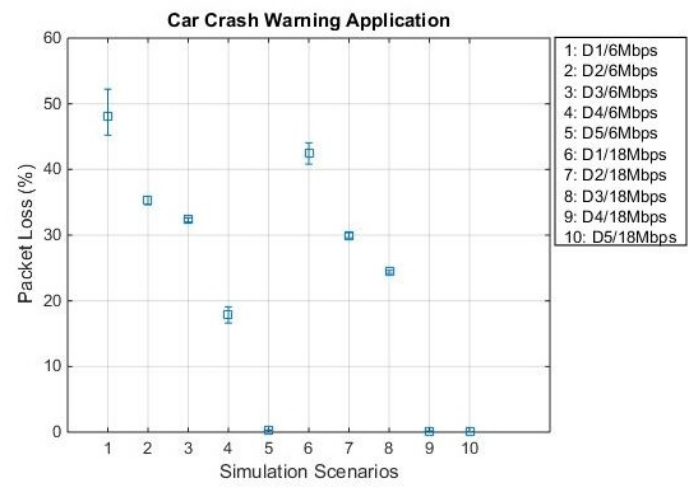

Fig. 4. Packet Loss for Car Crash Warning Appication

For both applications, different bit rates also affect the VANET V2I applications and specifically the Packet Loss. For 972 vehicles, Packet Loss is $89 * 10-3 \%$ for $6 \mathrm{Mbps}$ and $40 * 10-3 \%$ for $18 \mathrm{Mbps}$. Comparing with the Car Crash, in the Spot Weather application more vehicles receive the spot weather impact warning messages for the conditions of the highway network with lower values for high node densities.

Finally, when the number of vehicles increases the Packet Loss decreases because more and more vehicles receive the warning messages after an event. As a "global" warning message sent out to all vehicles within range, the Weather Warning Application is a more objective metric for assessment of the V2I robustness. The Car Crash Warning Application is an 'event-based' message sent out not periodically but under specific conditions.

Fig. 6 and 7 demonstrate how different applications and densities of vehicles influence the Total Busy Time for both applications. In this scenario, the different types of vehicles affect the mobility model because there is a higher delay due to the vehicle's varying dimensions.

As the number of nodes increases, the Total Busy Time increases as well, for all warning messaging applications. Comparing the warning messaging applications, the results depend on all the above parameters and as the number of vehicles increases, the value of Total Busy Time increases too for the Car Crash Warning Application.

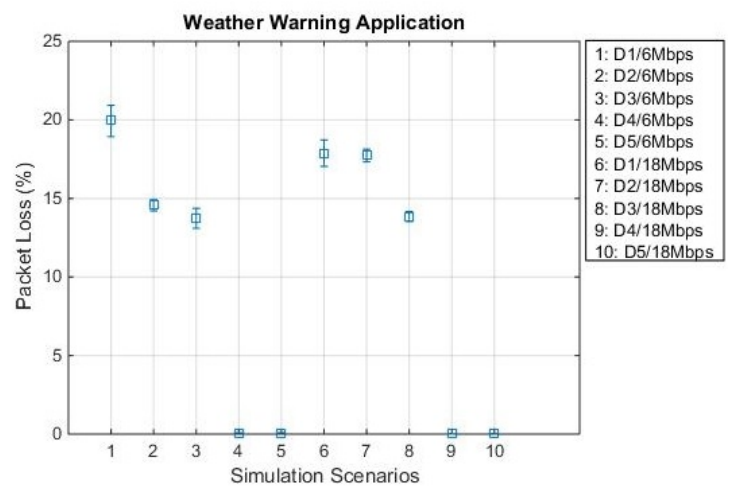

Fig. 5. Packet Loss for Weather Warning Application 


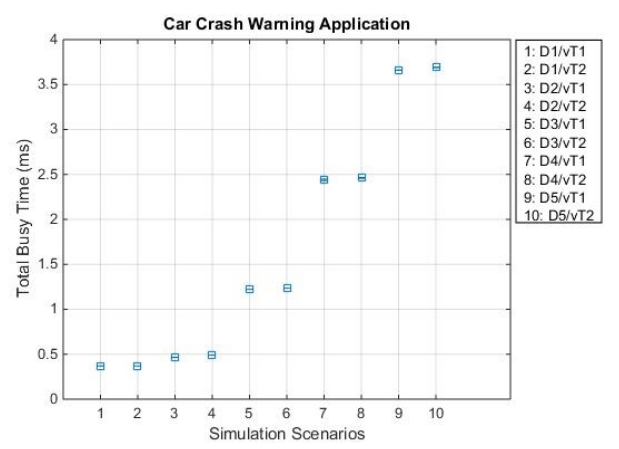

Fig. 6. Total Busy Time for Car Crash Warning Application

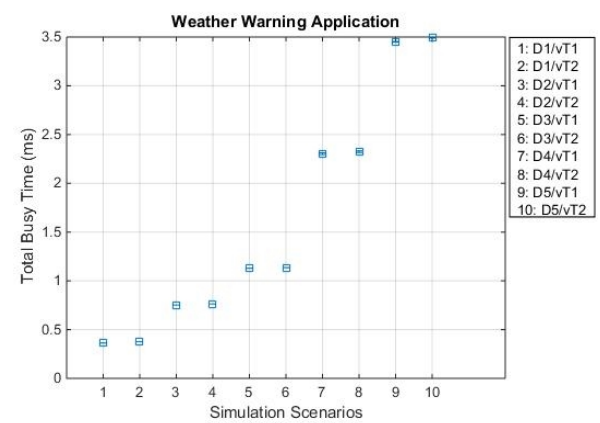

Fig. 7. Total Busy Time for Weather Warning Application

Moreover, for 324 vehicles and considering the different types of vehicles and applications, Total Busy Time is higher for the Car Crash than Weather Warning Application due to the fact that the duration of the crash is greater than the warning spots. For Car Crash, Total Busy Time for vehicleType1 is $1.22 \mathrm{~ms}$ and for vehicleType 2 is $1.23 \mathrm{~ms}$ because of the length of the vehicles. For Weather Warning Application, Total Busy Time is $1.12 \mathrm{~ms}$ and $1.13 \mathrm{~ms}$ for vTland vT2 respectively. When the number of vehicles doubles or triples the value of Total Busy Time doubles or triples too. Fig. 8 and 9 present how Total $\mathrm{CO} 2$ Emission is affected by different types of vehicles and densities. For both warning messaging applications, when the number of vehicles increases, the value of Total $\mathrm{CO} 2$ Emission increases too. When the number of vehicles doubles or triples, the values of Total CO2 Emission double or triple too.

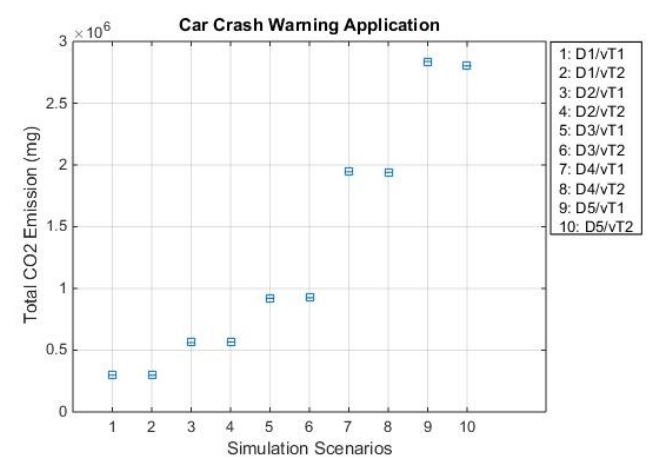

Fig. 8. Total $\mathrm{CO}_{2}$ Emissions for Car Crash Warning Application

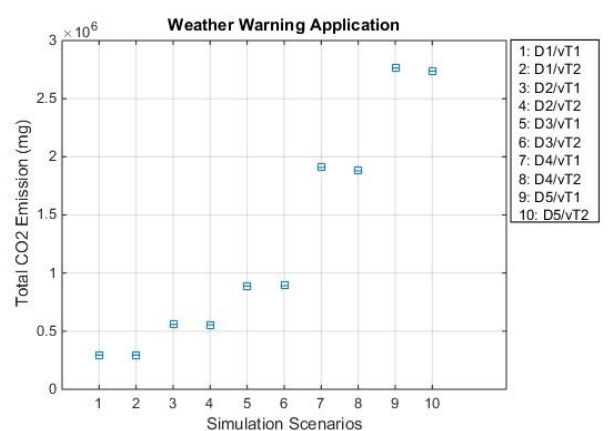

Fig. 9. Total $\mathrm{CO}_{2}$ Emissions for Weather Warning Application

\section{CONCLUSION}

This paper has studied how different applications can be generated in a VANET simulation environment for V2I communication with the RSUs. The paper has presented an analysis to determine RSU dimensioning in a highway taking into account the distance-dependent deterministic path losses as well as scattering losses. Excess path loss has been considered to follow the log-normal distribution and antenna heights are considered for the T-R separation distance. A threshold with a specific fade margin provides the required number of RSUs. The deployment of the RSU is such that for the bit rates and packet lengths of interest, the SNR threshold is sufficient to meet all essential requirements, for variable vehicles heights and velocities ranging up to $35 \mathrm{~m} / \mathrm{s}$.

We have investigated the impact of vehicle density, type of vehicles and different applications such as car crash and spot weather impact warning messaging on VANET performance evaluation. Realistic scenarios in a highway with real vehicle routes have been studied comparing scenarios with two different MAC Bit rates. In conclusion all the metrics are affected by vehicle density. VANET network metrics also affected by different MAC Bit rate and mobility model metrics by different vehicle type.

\section{FUTURE WORK}

Future work includes scenarios with higher number of vehicles, multiple vehicle crashes, RSU deployment for vehicle to LTE communication, a comparative study with V2I communication with RSU (802.11p) and implementation of other applications regarding the warning services of VANETs. LTE operates at a much lower frequency than 802.11 , with different bandwidth and system design. This comparison will allow us to draw conclusions about the efficiency of each wireless technology with regard to service delivery, taking into consideration the different frequency bands, Tx powers, sensitivity levels and modulation-coding schemes involved. In addition, the impact of setting specific thresholds for RSU deployment on the downlink bit rate, and therefore on the average number of users (vehicular nodes) that can be serviced by each RSU, will be investigated, for various pre-set bit rates per user. Generic text (warning messages) applications will be considered with little demand for bandwidth, and progressively more demanding applications will be added to our research plan. 


\section{REFERENCES}

[1] C. Manasseh and R. Sengupta, "Middleware to enhance mobile communications for road safety and traffic mobility applications", IET Intelligent Transport Systems, 4(1):24, 2010.

[2] IEEE 802.11p, "Draft standard for information technology telecommunications and information exchange between systems - local and metropolitan area networks - specific requirements: Wireless access in vehicular environments", IEEE P802.11p/D9.0, September 2009.

[3] IEEE Std 802.11p-2010, "IEEE Standard for Information technologyLocal and metropolitan area networks- Specific requirements- Part 11: Wireless LAN Medium Access Control (MAC) and Physical Layer (PHY) Specifications Amendment 6: Wireless Access in Vehicular Environments".

[4] C. S. Lin, B. C. Chen, and J. C. Lin, "Field test and performance improvement in IEEE $802.11 \mathrm{p} \mathrm{V} 2 \mathrm{R} / \mathrm{R} 2 \mathrm{~V}$ environments", in IEEE International Conference on Communications, IEEE Vehicular Networks \& Applications Workshop, Cape Town, South Africa, May 2010.

[5] M. Wellens, B. Westphal, and P. Mahonen, "Performance evaluation of IEEE 802.11-based WLANs in vehicular scenarios", in IEEE 65th Vehicular Technology Conference, April 2007, pp. 1167-1171.

[6] L. Cheng, B. E. Henty, R. Cooper, and D. D. Stancil, "A measurement study of time-scaled $802.11 \mathrm{a}$ waveforms over the mobile-to-mobile vehicular channel at $5.9 \mathrm{GHz}$, IEEE Communications Magazine, vol. 46, pp. 84-91, May 2008.

[7] D. Jiang and L. Delgrossi, "IEEE 802.11p: Towards an international standard for wireless access in vehicular environments," in IEEE 67th Vehicular Technology Conference, May 2008, pp. 2036-2040.

[8] D. Carona, A. Serrador, P. Mar, R. Abreu, N. Ferreira, T. Meireles, J. Matos, and J. Lopes, "A 802.11p prototype implementation", in Intelligent Vehicles Symposium (IV), 2010 IEEE, La Jolla, CA, USA, June 2010.

[9] K. Y. Ho, P. C. Kang, C. H. Hsu, and C. H. Lin, "Implementation of WAVE/DSRC devices for vehicular communications", in Computer Communication Control and Automation (3CA), 2010 International Symposium on, vol. 2, May 2010.

[10] A. Paier, R. Tresch, A. Alonso, D. Smely, P. Meckel, Y. Zhou, and N. Czink, "Average downstream performance of measured IEEE 802.11p infrastructure-to-vehicle links", in 2010 IEEE International Conference on Communications (ICC) Workshops, pp.1 -5.

[11] W. C. Jakes (Ed.), Microwave mobile communications. New York, NY: Wiley Interscience, 1974.

[12] D. W. Matolak, "Channel Modeling for Vehicle-to-Vehicle Communications", IEEE Communications Magazine (special section on Automotive Networking), vol. 46, no. 5, pp. 76-83, May 2008.

[13] D. W. Matolak, Q. Wu, and I. Sen, "5 GHz Band Vehicle-to-Vehicle Channels: Models for Multiple Values of Channel Bandwidth", IEEE Trans. Vehicular Tech., vol. 59, no. 5, pp. 2620-2625, June 2010.

[14] 3GPP TS36.104 version 11.8.2. R11.

[15] P. Vieira, M. P. Queluz, and A. Rodrigues, "LTE Multi Antenna Bit Rate Expectation for Urban Macro-cell Networks", Proc of 7th IberoAmerican Congress on Sensors (IBERSENSOR 2010), Lisbon, Portugal, Vol. 1, November, 2010.

[16] P. Vieira, M. P. Queluz, and A. Rodrigues, "LTE Spectral Efficiency using Spatial Multiplexing MIMO for Macro-cells", In 2nd IEEE International Conference on Signal Processing and Communication Systems, December 2008.

[17] I. Z. Kovacs, K. I. Pedersen, J. Wigard, F. Frederiksen, and T. E. Kolding, "HSDPA Performance in Mixed Outdoor-Indoor Micro-cell Scenarios" IEEE 17th International Symposium on Personal, Indoor and Mobile Radio Communications, pp. 1-5, September 2006.

[18] J. Seybold, Introduction to RF Propagation. Hoboken, NJ: Wiley Interscience, 2005.

[19] T. Rappaport, Wireless Communications: Principles \& Practice. Upper Saddle River, NJ: Prentice Hall, 1999.

[20] V. Shivaldova and C. F. Mecklenbräuker, "Real-world measurementsbased evaluation of IEEE $802.11 \mathrm{p}$ system performance", 5th IEEE
International Symposium on Wireless Vehicular Communications (WiVeC), pp. $1-5,2013$.

[21] M. Boban, R. Meireles, J. Barros, O. Tonguz, and P. Steenkiste, "Exploiting the height of vehicles in vehicular communication", IEEE Vehicular Networking Conference (VNC), pp. 163 - 170, 2011.

[22] A. Paier, D. Faetani, and C. F. Mecklenbräuker, "Performance evaluation of IEEE $802.11 \mathrm{p}$ physical layer infrastructure-To-vehicle real-world measurements", in Proceedings of ISABEL 2010, Rome, Italy, November 2010.

[23] G. Charalampopoulos and T. Dagiuklas, "Performance Evaluation of VANETs with Multiple Car Crashes in Different Traffic Conditions", 7th International Working Conference on Performance \& Security Modelling and Evaluation of Cooperative Heterogeneous Networks (HET-NETs) workshop, Ilkley, United Kingdom, 2013

[24] JOSM - Java OSM Editor, https://josm.openstreetmap.de.

[25] OpenStreetMap, http://www.openstreetmap.org.

[26] S. Grafling, P. Mahonen, and J. Riihijarvi, "Performance Evaluation of IEEE 1609 WAVE and IEEE 802.11p for Vehicular Communications", International Conference on Ubiquitous and Future Networks, pp. 344348, 2010.

[27] Simulation of Urban Mobility (SUMO), http://sumo.dlr.de/wiki/Main_Page.

[28] Veins, The open source vehicular network simulation framework, http://veins.car2x.org.

[29] A. Serageldin and A. Krings, "The Impact of Redundancy on DSRC Safety Application Reliability under Different Data Rates", in 6th International Conference on New Technologies, Mobility and Security, 2014.

[30] J. Santa, F. Perenguez, J. C. Cano, A. F. Skarmeta, C. T. Calafate, and P. Manzoni, "Comprehensive vehicular networking platform for V2I and V2V communications within the walkie-talkie project", International Journal of Distributed Sensor Networks, 2013. 